\title{
CONTRIBUTION DE LA "VICTIMOLOGIE" AUX SCIENCES CRIMINOLOGIQUES
}

\author{
P. Cornil
}

Le problème n'est pas nouveau. Le rôle joué par la victime dans l'infraction pénale est à l'origine d'importantes dispositions de nos Codes pénaux. Les criminologues envisagent - implicitement ou explicitement - l'attitude de la victime parmi les facteurs qui influencent l'auteur du crime. Les pénologues ont cessé de croire qu'ils pouvaient limiter leur action à la persone du délinquant. Ils se préocupent de l'influence du milieu social, et notamment de la situation de la victime.

Et pourtant, si le sujet n'est pas neuf, depuis une dizaine d'années il a pris une importance croissante. Eclairé sous un jour nouveau, il est susceptible d'apporter une contribution originale au problme pénal.

Deux hommes me paraissent avoir participé à cette évolution d'une façon particulièrement marquante.

H. von Hentig, par la publication en 1948 de son volume "The Criminal and his Victim", a exposé, dans les 65 dernières pages de cet ouvrage, une documentation abondante et un ensemble de conceptions, parfois simplement esquissées, sur le rôle de la victime dans l'infraction. Il y montre avec force que l' opposition légale entre l'auteur et la victime est beaucoup plus complexe que les catégories sommaires établies par le droit pénal (Bibliographie n..$^{\circ} 3$, p. 383).

Les auteurs qui ont traité ensuite ce même sujet ont puisé à la source de von Hentig et lui ont rendu hommage. 
Avant von Hentig on trouve, il est vrai, des aperçus intéressants sur la question, notamment chez E. Sutherland (v. bibl. n. ${ }^{0}$ 1), chez Et. De Greeff (bibl. n. ${ }^{\circ}$ 2) et chez Seeling (bibl. n. ${ }^{\circ} 4$ ), mais la question y apparait incidemment et n'est pas le sujet central de ces études.

Ce fut le mérite de B. Mendelssohn, en 1956, d'attirer violemment l'attention sur ce sujet. Il le fit en un article publié $\in \mathrm{n}$ anglais et en français (.$^{\circ}{ }^{6}$ ) dans lequel il tenta d'ouvrir une voie nouvelle en étudiant "la victime en tant que victime". Cette science, qu'il baptise du nom de "victimologie" serait distincte de la criminologie. Mendelssohn préconise la création d'Instituts de recherches et de chaires de victimologie dans les Universités. Il demande en outre l'organisation de Congrès de victimologie (art. cité, conclusion). Enfin, dans une nouvelle étude qui parait dans ce numéro, Mendelssohn invente d'autres néologismes et oppose le "victimel" au criminel, la "victimalité" à la criminalité.

Cette position extrême montre à quel point son auteur est convaincu de la nouveauté et de l'importance de son sujet, ainsi que de son originalité par rapport aux disciplines existantes, et notamment à la criminologie. Son affirmation catégorique a eu le mérite, par son excés même, d'orienter des pénalistes et des criminologues vers un domaine peu exploré et riche de possibilités. La dénomination de "victimologie" est commode pour la facilité de l'exposé. Je ne crois pas, cependant, et j'essaierai de la montrer, qu'il soit utile ni opportun de constituer, sous cette appellation, une discipline autonome au sein de l'ensemble des sciences pénales.

Voyons donc de plus près comment ce problème peut être étudié. Il est manifeste que le bon sens populaire considère le criminel et la victime comme essentiellement différents l'un de l'autre. Comme le dit Ellenberger, il oppose criminel et victime comme noir et blanc. La conception juridique ne s'éloigne que fort peu de cette façon de voir. Elle continue, sauf dans certains cas extrêmes, à opposer le criminel coupable et la victime innocente (H. Ellenberger, n. ${ }^{\circ} 5$ ). 
Et cependant, les découvertes de la psychologie profonde et les recherches criminologiques démontrent aujourd'hui clairement l'insuffisance de cette position pénale qui reste centrée sur la notion d'auter diamétralement opposée à celle de la victime. Comme le dit von Hentig, les motifs de l'action ne se créent pas dans le vide. Ils sont suscités par des influences extérieures, en même temps qu'ils provoquent l'action de l'auteur. Or. l'une de ces influences extérieures, et non des moindres, est fréquemment la personnalité ou l'attitude de la victime (n. ${ }^{\circ}$, p. 397).

\section{Définitions et limites.}

Damandons-nous d'abord ce qu'il faut entendre par victime (néerlandais: "Slatchtoffer"; allemand: "Opfer"; anglais: "Victim"). Le sens originaire de ce mot, qui est plus accentué encore, dans sa traduction néerlandaise ou allemande, est de caractère religieux. On vise le sacrifice d'une persone ou d'un animal à la divinité. Le sens moderne de ce mot est cependant beaucoup plus étendu: Litré considère notamment comme victime "celui qui est sacrifié aux intérêts, aux passions d'autrui". Selon une autre définition, c'est "la personne qui succombe, la personne qui subit les conséquences d'un acte, d'un fait, d'un accident" (Larousse).

Comment faut-ll comprendre ce mot en droit pénal? Peut-on dire qu'il s'agit tout simplement du préjudicié, de la personne lésée par l'infraction? Le professeur Schultz, dans une étude récente sur les relations entre l'auteur et la victime, (n. $\left.{ }^{\circ} 11\right)$ limite son examen aux infractions qui comportent des relations entre individus (Beziehungsverbrechen), excluant ainsi les autres infractions, celles qui lèsent une règle de droit, par exemple la haute trahison, et même des infractions contre la propriété, p. ex. le vol. J'estime que cette limitation est arbitraire et qu'il faut prendre ce concerpt dans un sens plus large car, comme le laisse entendre von Hentig, la victime peut être aussi bien un individu que la communauté (op. cit., p. 450). Presque toute infraction fait une victime soit directement, soit indirectement, et Schultz souligne lui-même que l'attitude insouciante ou l'inattention du propriétaire d'un 
objet peut en favoriser le vol (2). Il n'y a donc pas de raison d'exclure ce genre d'infraction du domaine de nos investigations.

Nous en arrivons ainsi à considérer que la victime n'est pas nécessairement un être vivant. Elle peut aussi être un bien matériel.

Caractère antipathique de cette étude.

Depuis que j'ai eu l'attention attirée sur ce problème, je me suis aperçu très rapidement de la méfiance et des critiques qu'il suscite, surtout de la part des personnes qui n'ont pas réfléchi au problème pénal, et même de certains spécialistes. En effet, mettre en relief le rôle de la victime d'une infraction aura parfois pour effet d'imputer à la victime une partie de la faute et, par conséquent, de diminuer la responsabilité de l'auteur. C'est pourquoi on s'inquiète de ce nouvel effritement de la répression. Ce sera, me dit-on, encore un moyen d'excuser les délinquants et de les traiter avec une indulgence excessive.

Par ailleurs, il faut reconnaitre que les critiques formulées à l'audience au sujet de la conduite de la victime sont déplaisantes, surtout lorsqu'il s'agit de la victime d'un homicide qui n'est donc pas présente pour se défendre. En Cour d'assises, il arrive que la défense prononce un véritable réquisitoire à charge de la mémoire de la victime, appelant une réplique véhémente et indignée de la partie civile.

Enfin, on reproche aux études sur le rôle de la victime d'aboutir à jeter le trouble dans les esprits car, comme je le

(2) La Cour d'Appel de Bruxelles vient de rendre un intéressant arrêt selon lequel l'abandon d'une voiture automobile sur la voie publique, sans fermer les portières à clef et la clef de contact restant à sa place, constitue une faute permettant de rendre le propriétaire de la voiture civilemet responsable du dommage causé à un tiers, à la suite d'une collision provoquée par un inconnu qui s'est emparé de sa voiture (v. Appel Bruxelles, 17 décembre 1958, J. T. 1er février 1959, p. 78 ). 
disais plus haut, le sens commun a horreur de toute conception qui ne distingue pas nettement entre le criminel et le non-criminel, comme entre le malade mental et le sain d'esprit (v. Ellenberger, n. ${ }^{\circ}$ 5, p. 106).

Quelle que soit la valeur de ces objections, elles ne peuvent cependant pas nous empêcher de nous pencher sur ce problème d'une façon objective.

La victime et la loi pénale.

Comme von Hentig le montre, il y a rarement une opposition absolue entre l'auteur de l'infraction et sa victime, parce qu'il y a toujours une action réciproque, et parfois c'est le hasard lui-même qui détermine quelle sera la victime. C'est ainsi que les coups réciproques dans une rixe peuvent avoir des suites graves chez l'un des participants, qui deviendra de ce fait victime (Elleberger, n. ${ }^{0}$ 5, p. 105). Lorsque deux personnes décident de se batre eu duel, elles sont mues toutes deux par la même intention et la même volonté de faire usage d'ue arme déterminée pour se combattre. Selon le Code pénal belge, ce fait à lui seul est punissable. L'usage des armes contre un adversaire en duel, même sans qu'il en résulte un homicide ou des blessures, est frappé d'une sanction pénale, mais, en outre, celui qui, plus adroit ou plus chanceux que l'autre, blesse ou tue son adversaire, sera frappé d'une peine plus grave. A ce moment, l'un des deux participants devient la victime.

Dans certains cas, il peut y avoir doute: on ne sait pas si on a affaire à un auteur ou à une victime.

Il y a quelques années, j'ai connu dans la province de Luxembourg l'incident suivant: un incendie détruisit complètement ūne maison de paysan. Ce bâtiment avait été assuré peu de temps auparavant. En outre, certains indices parurent suspects, notamment le fait que la basse- cour avait été mise en sécurité chez un voisin. Le propriétaire fut arrêté et, relâché après quelques semaines de détention, il a touché la prime d'assurance. On ignore encore s'il a été l'auteur ou la victime de cet incendie. 
Parfois, on démasque une fausse victime: pour dissimuler un vol ou un détournement dont il était l'auteur, un propriètaire simule une attaque à main armée et fait croire qu'il a été dévalisé (v. von Hentig, n. ${ }^{0} 3$, p. 401).

La bigamie est, en Belgique, un crime punissable de réclusion (art. 391, C. P.). Quelle en est la victime? On peut se livrer à des plaisanteries faciles sur le sort de l'homme qui a épousé plusieurs femmes. Il est piquant de lire dans les mémoires de Lewis Lawes que le bigame n'est presque jamais un homme beau, entreprenant ou intelligent. Il est le plus souvent si efféminé que Lawes se demande s'il n'a pas été plus séduit que séducteur (Lawes - "Life and Death in Sin-Sing" p. 94). Il ajoute que, en général, les femmes des bigames demandent toutes à leur rendre visite, mais le règlement s'y oppose. Von Heting interprète cette défense comme une mesure de protection de ces pauvres épaves $\left(\mathrm{n}^{\circ} 3, \mathrm{p} .414\right.$, note 91 ).

La loi pénale ne reconnait qu'exceptionnellement le rôle joué par la victime dans l'infraction en lui attribuant des effets particuliers. L'exemple le plus caractéristique est celui de la légitime défense. Celui qui comment un homicide ou qui porte des coups peut être justifié lorsqu'il a été l'objet d'une attaque de sa future victime et que sa réaction était le seul moyen dont il disposait pour se défendre (C. P. belge, art. 416). En pareille hypothèse, l'attitude de la victime fait disparaitre complètement l'infraction. La loi belge prévoit, en outre, des causes d'excuse et tout d'abord la provocation, qui diminue la peine lorsque l'homicide, les blessures et les coups ont été provoqués par des violences graves envers les personnes (art. 413). Cette atténuation de peine est donc, elle aussi, fondée sur comportement de la victime. Il en sera de même lorsque l'auteur d'un homicide, de blessures ou de coups a surpris son épouse et son complice en flagrant délit d'adultère (art. 413 du C. P.).

Pour certaines infractions les poursuites ne sont engagées que sur plainte de la victime. Il en est ainsi notamment dans la plupart des infractions qui portent atteinte à l'honneur (art. 450 du C. P.). On estime qu'il appartient à la vic- 
time elle-même d'apprécier l'opportunité des poursuites. Il en est de même en matière d'adultère, où la poursuite n'a lieu que sur plainte de l'époux, qui pourra même arrêter l'effet de la condamnation en consentant à reprendre son épouse (art. 387 à 390$)$.

L'attitude de la victime peut-elle être une circonstance atténuante que permettra au juge de diminuer le peine? Le Code pénal belge, comme le Code français, ne définit pas les circonstances atténuantes et les laisse à l'appreciation du juge. (C. P. art. 79 et suivants). Elles peuvent donc être invoquées sans aucune motivation et forment une disposition passe-partout, susceptible notamment de compenser le rôle joué par la victime dans l'infraction. Du reste, dans son traité, Adolphe Prins cite notamment, parmi les causes d'atténuation de la peine "les séductions auxquelles le délinquant a été en butte", reconnaissant ainsi d'une façon explicite que la conduite de la victime peut être prise en considération (Science pénale et droit positif - N. $\left.{ }^{\circ} 401\right)$. Le Code pénal suisse le prévoit en termes exprès lorsqu'il énumère les circonstances atténuantes. On trouve parmi celles-ci: “Lorsqu'il (l'auteur) aura été induit en tentation grave par la conduite de la victime" (C. P. suisse - art. 64, par. 5).

Dans la plupart des législations, le consentement de la victime n'est pas reconnu comme valable, à moins que ce consentement ne supprime un élément essential de l'infraction comme, par exemple, dans le cas de prétendu viol d'une personne majeure et normale. Cependant, le Code pénal suisse prévoit une atténuation de peine à l'égard de l'auteur d'un meurtre commis à la demande de la victime. L'article 114 de ce code déclare en effet: "Celui qui, sur la demande sérieuse et instante d'une persone, lui aura donné la mort, sera puni d'emprisonnement".

D'une façon générale, on voit donc que les Codes pénaux ne tiennent compte de l'attitude de la victime que dans de rares cas. Il est vraisemblable, cependant, que dans l'application de la loi pénale belge le recours aux circonstances atténuantes soit fréquemment motivé - même si cela résulte pas ex- 
plicitemment du jugement - par certains aspects de l'attitude de la victime.

Le point de vue criminologique.

La conception juridique que nous venons d'esquisser est basée sur une psychologie simplifiée et qui manque de nuances. En réalité, le problème est beaucoup plus complexe et il doit être examiné également du point de vue criminologique.

Tout d'abord, on a relevé l'intérêt criminalistique de l'examen de la victime d'un meurtre. Selon le moyen employé pour commettre l'homicide, on peut découvrir la profession de son auteur: un militaire ou un braconnier se serviront d'une arme à feu; la hache et aussi la méthode de dépeçage d'un cadavre révèleront l'intervention d'un boucher; l'homicide commis par absorption de produits médicaux fera rechercher le coupable parmi les médecins ou les infirmières. Mais il y a plus: presque toujours, il existe des relations entre le criminel et la victime, antérieurement au crime. Ces relations peuvent être de nature très diverse. Elles peuvent se placer sur lo plan professionnel ou être d'ordre privé; être inspirées par l'inimitié, par l'amour ou par la haine. Le criminel qui frappe au hasard est rare parmi les individus normaux. Presque toujours il existe une relation criminel-victime. Cette relation peut d'ailleurs être plus ou moins "objective", par exemple lorsque'elle vise la fortune de la victime, les valeurs qu'elle transporte ou qu'elle détient (v. Ellenberger, n. ${ }^{0} 5$, p. 114. contra, Sutherland, n. ${ }^{\circ} 1$, a, p. 20-21, qui estime que les infractions contre les biens sont généralement commises à l'égard đ'étrangers). Cette relation criminel-victime peut être apparente au premier abord. Elle peut aussi être mal comprise ou déformée par l'observateur superficiel. Sa mise en évidence peut constituer un élément essential dans la recherche de l'auteur e dans l'explication de son acte. L'oeuvre d'Etienne De Greeff est imprégnée de l'importance et de la complexité de cette relation criminel-victime, spécialement dans le crime passionnel (v. De Greeff - n. ${ }^{0} 2$ - a et b, pp. 390 ss.). 
Du point de vue criminologique, l'étude des victimes d'homicides nous montre que certaines professions sont spécialement exposées aux attentats. Un exemple qui est d'actualité est celui du chauffeur de taxi. Par ses conditions de travail, ce chauffeur est particulièrement exposé aux agressions. Des clients se font conduire dans un endroit désert; le chauffeur leur tourne le dos et peut facilement être attaqué par derrière. Les agresseurs peuvent alors se débarrasser de lui après l'avoir délesté de son argent et ils peuvent utiliser la voiture pour s'enfuir. Ce genre d'attentat est relativement fréquent. Il y a peut d'années, deux anormaux évadés de l'établissement de défense sociale de Tournaiont commis un crime de cette espèce pour faciliter leur fuite.

On recherche les moyens préventifs pour mettre fin à ces attentats. Le placement de signaux d'alarme fonçtionnant automatiquement semble avoir déjà donné certains résultats. Il faudrait aussi trouver le moyen d'éviter que les chauffeurs de taxi ne transportent sur eux des sommes d'argent importantes.

D'autres professions exposées sont celles d'employé de banque, de postier ou, en générel, d'agent présposé au transport de sommes d'argent. Les vols avec violences de la paie du personnel d'une entreprise sont assez fréquents, notamment aux Etatis-Unis et en Angleterre. Ils se produisent d'ailleurs de temps à autre chez nous. Il y a quelques moins, un attentat a été commis avec homicide dans une banque de Dinant. Les meurtriers sont toujours en fuite.

On s'émeut, en Angleterre, du grand nombre de ces attentats contre les transports de sommes d'argent. Selon une déclaration faite récemment au Parlement, dans le seul district métropolitain de Londres, il y eut 31 attentats de ce genre en 1957 et 35 pendant les 10 premiers moins de l'année 1958.

Parmi les mesures envisagées pour diminuer le nombre de ces vols, on propose de modifier une série de lois dont la première remonte à 1831 (Guillaume IV, chap. 37 du 15 oct. 1831), connues sous le nom de "Truc Acts". Ces lois, qui trouvent leur équivalent dans d'autres pays, et notamment en 
Belgique (Loi du 16 aout 1887 sur les salaires, art. 1), défendent le paiement des ouvriers autrement qu'en monnaie et en billets de banque. Elles avaient pour objet de mettre fin au paiement des salaires en nature connu sous le nom de truck-system. La rigueur de ces textes - dépassant leur objectif premier - s'oppose au paiement des salaires par chèque ou par mandat postal. Un projet de loi déposé en 1958 (Wages Bill, - 12 nov. 1958) tend à autoriser ces modes de paiement. La réalisation de cette réforme se heurte copendant à diverses difficultés d'ordre pratique: les banques ferment leurs guichets à 3 heures de l'après-midi. Comme beaucoup de familles de salariés ont l'habitude de faire leurs achats le jour de la paie, les commerçants craignent que de nombreux clients leur demandent d'encaisser des chèques à la fin de l'après-midi, les obligeant à accumuler des sommes importantes dans leur caisse, attirant ainsi sur eux l'attention des malfaiteurs qui s'attaquent aujourd'hui aux transporteurs de fonds. Une autre difficulté pratique surgirait pour les banques, dans l'hypothèse ou elles prolongeraint les heures d'ouverture des guichets : elles devraient, à certains jours, satisfaire une nombreuse clientèle, desireuse d'obtenir son argent au même moment (renseignements fournis par M. Hugh Klare - Secrétaire de la Howard League for Penal reform - 7 janvier 1959). C'est néanmoins dans ce sens que doit s'orienter la prévention des attaques à main armée contre les transporteurs de sommes importantes destinées au paiement de salaires.

J'ai cité ces professions à titre d'exemples particulièrement actuels; les auteurs en citent plusieurs autres, et notamment la profission de prostituée.

Certaines situations personnelles ou familiales suscitent également des homicides et attirent l'attention sur le rôle joué par la victime. Il y a notamment la situation pénible de la femme vieillissante qui cherche à sortir de son isolement (Werner, n. ${ }^{\circ}$ 7, p. 3).

Les Bruxellois de ma génération se souviennent de ce crime qui fit tant de bruit quelques années avant la guerre: un jeune bourgeois s'était rendu à Paris avec une amie sans scru- 
pule. Manquant d'argent, il était devenu l'amant d'une dame riche, d'âge mur. A l'instigation de son amie, il la tua pour la dévaliser. L'imprudence naive de sa victime avait favorisé cette entreprise criminelle (affaire N..., plaidée par Me Braffort).

Plusieurs auteurs signalent le cas de familles où le père tyrannique et alcoolique tombe victime de sa femme et parfois de ses enfants (voir E. Werner - n. $.^{0} 7-$ pp. 2 à 5). Je me souviens d'avoir connu en 1934, à la prison-école d'Hoogstraten, deux jeunes gens condamnés à des peines de travaux forcés du chef de parricide. Ils avaient, avec l'aide de leur mère - également condamnée - tuè le père brutal et alcoolique. C'étaient, au demeurant, deux jeunes gens intelligents et ouverts, qui se sont complètement reclassés à leur libération.

Il y a quelques semaines, les journaux ont relaté un drame familial qui s'est produit à Anvers et qui a trouvé son épilogue devant le tribunal correctionnel. Un hombre, âgé de 55 ans, a été l'objet d'une tentative de meurtre commis par sa femme et par un jeune homme qui voulaint épouser une des filles de la victime. Le père, violent et despotique, rendait la vie impossible à sa famille. Deux autres filles avaient déjá fui la maison paternelle pour se marier malgré la volonté de leur père. Un soir, son épousse avaint essayé de l'empoisonner, en mêlant de l'arsenic à son café. Le mari avait refusé la boisson dont il trouvait le gout suspect. Sa femme alla chercher le futur gendre et le jeune homme assomma son futur beau-père qui fut assez sérieusement blessé, mais parvint cependant z̀ mettre en fuite son agresseur. Bien que les accusés eussent avoué qu'ils avaient l'intention de jeter le corps de la victime dans les eaux du rempart, l'épouse ne fut condemnée qu'à un an de prison et son complice s'en tira avec une peine de cinq mois. Il faut croire que le tribunal a largement tenu compte de la conduite de la victime:

On pourrait citer de nombreux cas de cette espèce. La presse en rapporte fréquemment et l'examen des dossiers judiciaires en révèle un bon nombre. 
On a relevé, à diverses reprises, que certains individus paraissent poursuivis par la malchance et sont, plus fréquemment que d'autres, victimes d'accidents ou d'attentats. Cette constatation a été faite particulièrement dans le domaine des accidents du travail. Il semble que certaines persones soient nées malchanceuses ou maladroits. "Dans les jardins zoologiques, on sait qu'il faut éliminer certains sujets du travail avec les animaux car ils se font immanquablement blesser par eux". Ellenberger, qui rapporte cette constatation, déclare que ce domaine psychologique n'a guère été exploré jusqu'a présent (voir Ellenberger, n. ${ }^{\circ}$ 5, p. 113).

D'une façon générale, on est surpris de voir combien la naïveté et la crédulité du public peuvent être expliquées facilement.

Les spécialistes de la publicité commerciale savent combien la répétition de certaines affirmations laudatives peut exercer une influence sur le lecteur des annonces. En particulier dans le domaine médical et pharmaceutique, le malade et même celui qui se croit atteint de quelque affection imaginairc se laisset aisément persuader de l'efficacité d'un traitement sans prendre la précaution de vérifier le sérieux et le fondement scientifique de la méthode préconisée. Il en est ainsi particulièrement lorsque le malade perd confiance dans la medicine ou se croit abandonné par le corps médical. L'individu inquiet se saisit de la moindre promesse et se laisse guider par toute leur d'espoir.

De même, l'appât de gains même illusoires attire un public nombreux vers les loteries, les spéculations hasardeuses et facilite par cette disposition d'esprit la tâche de l'escroc (voir article de Me. Calewaert). Un exemple typique de la facilité avec laquelle le lecteur d'une annonce se laisse suggestionner même sans qu'aucune promesse ne lui soit faite est rapporté par Mlle Marx dans un écho récent qu'elle intitule "A propos de la victimologie". Une annonce parait dans un journal de New York: "Apportez-moi un dollar, Smith, 47e rue, n. ${ }^{\circ}$ ". Le lendemain, en même place, une annonce deux fois plus grande: "Vous avez encore jusqu'à demain pour 
m'apporter un dollar, Smith, 47e rue, n. ${ }^{\circ}$ ". Le troisième jour, l'annonce est trois fois plus grande que la première: "Si vous n'avez pas apporté le dollar à mon bureau avant 4 heures de l'après-midi, il sera trop tard, Smith, 47 e rue, n..$^{\circ} 4$ ". Quelle était l'explication de ces annonces? M. Smith avait parié que cent personnes au moins donneraient suite à cette demande de paiement sans contre-partie. En fait, 1.600 personnes répondirent à cet appel (v. Yvonne Marx - "A propos de la victimologie" - Revue de Science Criminelle et de Droit pénal Comparé - 1958 - p. 244).

Il sembre bien qu'un nombre considérable de personnes, dans le désir vague et irraisonné d'obtenir un avantage quelconque, perdent tout sens critique et sont victimes de ce phénomène d'aveuglement partiel qu'Elleberger appelle "scotome", tache aveugle qui empêche la vision d'un point déterminé $\left(\mathrm{n} .^{0} 5, \mathrm{p} .110\right)$.

Cette absence de sens critique d'un grand nombre d'individus se trouve renforcée lorsque le sujet est dans une situation difficile qui paralyse toute réaction de sa part en l'exposant éventuellement à des manoeuvres de chantage. Celui qui a un besoin impérieux d'emprunter de l'argent se soumettra aux exigences de l'usurier qui le sort momentanément d'embarras.

Par ailleurs, un individu qui, sans avoir commis une véritable infraction, pourrait être compromis dans une poursuite judiciaire peut faire l'objet de manoeuvres de chantage. Le professeur Sutherland, dans un ouvrage écrit en 1937 d'après les memóires d'un voleur professionnel, relate deux exemples typiques de ces procédés. Il cite notamment une bande d'escrocs qui opérait dans les stations de métro de New York, aux environs de 1910. Ils recherchaient des homosexuels qu'ils s'efforçaient de surprendre dans une attitude compromettante, dans les lavatoires de ces stations; parfois même, un membre de la bande remplissait le rôle d'agent provocateur. Les escrocs exigeaient alors le paiement d'une forte somme d'argent, sous menace de dénonciation à la police. Bien entendu, 
la somme était payée et presque toujours la victime se gardait bien d'alerter l'autorité.

Une autre forme ingénieuse de chantage visait les déclarations d'impôts. Un escroc se présentait chez un particulier ou un commerçant, en se faisant passer pour un fonctionnaire des finances. Il affirmait qu'une fausse déclaration d'impôts avait été faite et exigeait le paiement d'une somme pour arrêter les poursuites. Cette manoeuvre réussissait presque toujours, ce qui prouve notamment que la fraude fiscale était fréquente et que l'escroc avait donc deviné juste. Pour s'assurer l'impunité, ces escrocs opéraient surtout le samedi, de façon à éviter toute vérification avant le lundi (v. Sutherland - n. ${ }^{\circ}$ 1, b. - pp. 79 à 81).

Dans les cas d'infractions caractérisées l'auter peut être amené à commettre un crime grave pour échapper à l'arrestation. Un cas classique est celui du voleur supris par le propriétaire ou par la police, qui va jusqu'à commettre un homicide pour s'enfuir (C. Werner, n. ${ }^{\circ}$ 7, p. 4).

Le Code pénal belge vise spécialement cette hypohèse et frappe de la peine capitale l'auteur d'un homicide commis pour assurer l'impunité du vol. (art. 475).

Werner cite aussi les cas du pédophile qui est amené à accomplir un homicide sur des enfants victimes de ses attentats, afin d'éviter que ceux-ci ne le dénoncent (n. ${ }^{0} 7$, p. 4).

Rôle actif de la victime.

Dans des nombreaux cas, on met en évidence que la victime consent tacitement à l'infraction, parfois même qu'elle y coopère ou qu'elle la provoque (v. von Hentig, n. ${ }^{\circ} 3$, pp. 419 et ss.). Ceci est particulièrement vrai dans bon nombre d'infractions contre les moeurs. La loi établit une présomption selon laquelle les mineurs en-dessous d'un âge déterminé soint nécessairement victimes de l'infraction. En fait, les enquêtes révèlent que certains victimes ont joué un rôle plus ou moins actif. On peut cependant admettre, du point de vue de la politique criminelle, l'opportunité de maintenir cette fiction léga- 
le qui présume la responsabilité de l'adulte même s'il a été l'objet de certains tentations.

Ce problème a été étudié particulièrement en ce qui concerne les meurtres. Dans un article récent, l'Américan Wolfgang a étudié un ensemble d'homicides commis à Philadelphie, du ler. janvier 1948 au 31 décembre 1952. Il s'agissait de 588 affaires dans lesquelles étaint impliqués 621 criminels. Dans 150 dossiers, soit $26 \%$ du total, la victime avait favorisé l'homicide, soit en usant la première de force physique, soit en montrant une arme, en portant des coups, etc... (Wolfgang, n. ${ }^{\circ} 8-$ pp. 1 à 11).

Il est difficile de se rendre compte exactement des circonstances dans lesquelles ces homicides ont été commis, mais il parait vraisemblable que, selon notre législation, on aurait $\mathrm{pu}$, dans un certain nombre de cas, invoquer l'excuse de la provocation.

Wolfgang a relevé le nombre de cas où les victimes avaient irigéré de l'alcool. Cette constation fut positive dans $69 \%$ des cas (n. ${ }^{\circ}$, p. 9; voir Verkko, cité par Schultz, n..$^{\circ} 11$, p. 176); voir aussi Rosenberg - Actes du 4e Congrès international de défense sociale - Milan 1956 - Vol. I, p. 695 et s., Milan Giuffré - 1957).

Il est plus curieux encore de constater qu'un grand nombre de ces victimes que avaient favorisé l'homicide étaien pourvues d'un casier judiciaire. Wolfgang montre que la présence de condamnations antérieures est plus fréquente chez les victimies qui ont favorisé le crime que chez les auteurs de crimes qui n'ont pas été favorisés par la victime (art. cité, p. 9). Il en arrive donc à conclure que c'est parfois le hasard qui détermine la victime et qu'il s'agit en réalité de deux délinquants potentiels. Cette constatation confirme une remarque que je faisais au début de cet exposé.

Traitement des délinquants.

Les constations que je viens de relever doivent exercer une influence sur les méthodes de traitement des délinquants. 
En effet, dès l'établissement du diagnostic, lorsqu'on étudie la personnalité du délinquant et son milieu social, ces investigations doivent porter à la fois sur l'auteur lui-même et aussi sur le milieu familial et social, qui comprend tout naturellement la personnalité de la victime elle-même. Cette constatation n'est pas neuve. Elle apparait très clairement dans les enquêtes faites par les juridictions pour mineurs et dans l'application des méthodes de probation.

Il en est de même, dans une certaine mesure, lors de l'examen de décisions de libération conditionnelle.

Bref, la pratique pénale et pénitentiaire nous déjà habitués à dépasser largement la personnalité de l'auteur de l'infraction pour nous préoccuper également de l'entourage de celui-ci et notamment de la victime. C'est ainsi, par exemple, que les conditions mises à un placement sous probation ou à une libération conditionelle doivent fréquemment tenir compte de la victime et parfois imposer à celle-ci certaines conditıons, notamment de ne pas résider au même domicile que l'auteur de l'infraction.

Ceci m'amène à terminer par l'enoncé de quatre conclusions générales qui visent à montrer dequelle façon la perspective "victimologique" peut s'insérer dans la technique de quatre branches des sciences criminologiques:

I. - La CRIMINOLOGIE étude l'ensemble des facteurs qui déterminent l'infraction. Parmi ceux-ci, l'attitude ou la situation de la victime est un élément important dont l'examen devrait être approfondi. Il n'y a cependant pas de raison décisive d'ériger cette recherche en discipline autonome.

II. - Le DROIT PENAL aborde ce problème sous l'angle d'une conception- simplifiée et superficielle de la psychologie. Il permet cependant au juge, par le recours aux circonstances atténuantes, de s'inspirer de conceptions plus nuancées.

III. - La POLITIQUE CRIMINELLE doit se préoccuper des faits révélés par la victimologie, pour éviter la 
création de situations criminogènes et afin d'entreprendre une action préventive efficace.

IV. - Le TRAITEMENT DES DELINQUANTS doit s'étendre à leur milieu social. Il peut éventuellement atteindre la victime de l'infraction.

\section{B I B L I O G R A P H I E}

1. - E. SUTHERLAND

a) Principles of Criminology - Chicago Lippincott - 1934 - p. 20-22.

b) The professional thief (annotated and interpreted by E. Sutherland) - The University of Chicago Press-Chicago - 1937.

2. - Et. DE GREEFF

a) La psychologie de l'assassinat - Rev. de Droit pénal et de Criminologie 1935 - pp. 153 et ss., 213 et ss., 357 et ss.

b) Introduction à la criminologie - 2e éd 1947 - sp. pp. 367 et 374 .

3. - H. VON HENTIG

The Criminal and his Victim - New Haven, York University Press - 1948.

4. - E. SEELIG

Lehrbuch der Kriminologie - Graz - 1951; trad. française Traité de Criminologie - Presses Universitaires de France 1956.

5. - H. ELLENBERGER

Relations psychologiques entre le criminel et la victime - Revue internationale de criminologie et de police technique - 1954 pp. 103-121.

6. - B. MENDELSOHN

a) The victimology - Etudes internationales de psycho-sociologie criminelle juillet-sept. 1958 - pp. 25-36; voir aussi Revue française de psychanalyse et Revue internationale de criminologie et de police technique $-1956-$ pp. 95 et ss.

b) La victimologie, science actuelle, Revue de Droit pénal et de Crimionologie avril 1959.

7. - E. WERNER

Das Opfer des Mordes - Kriminalistik 1956 - pp. 2-5.

8. - M. WOLFGANG

Victim prcipitated criminal homicide Journal of criminal law and criminology - May-June 1957 - pp. 1-11.

9. - W. SCHLUTER

Die Dummen werden nicht alle - Die Polizei Praxis n. ${ }^{\circ} 20$ - p. 295. 
10. - C. EHRLICH

Der Betrüger, sein Handwerkszeug und seine Opfer - Kriminalistik - Oktober 1957 - pp. 365-7.

11. - H. SCHULTZ

Kriminologiesche und Strafrechtliche Bemerkungen zur Beziehung zwischen Tater und Opfer - Revue pénale suisse 1958 pp. 171-191.

12. - D. REIFEN

Le délinquant sexuel et sa victime - Revue internationale de l'enfant - 1958 - pp. 110-124.

13. - C. R. SOUCHET

La victimologie - La Vie Judiciaire - 15 décembre 1958. 